\title{
Oostendorp, Rebekka (2016): Wohnstandortentscheidungen von Doppelverdienerhaushalten. Zum Zusammenwirken von Wohnen und Arbeiten in der polyzentrischen Stadtregion Köln/Bonn
}

\author{
Münster: LIT-Verlag. $=$ Schriften des Arbeitskreises Stadtzukünfte der Deutschen \\ Gesellschaft für Geographie 16, 11 Tab., 38 Abb., 256 S.
}

Michael Droß ${ }^{1}$

Online publiziert: 18. April 2017

(c) Springer-Verlag Berlin Heidelberg 2017

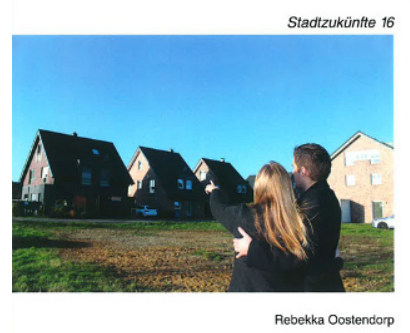

Wohnstandortentscheidungen von Doppelverdienerhaushalten Zum Zusammenwirken von Wohnen und Arbeiten in der
polyzantrischen Stadtregion Kön/ Bonn

LT

Bei einer Diskussionsveranstaltung über die Wohnungsmarktentwicklung in der Region München im Februar 2016 wurde deutlich, dass urbanes Wohnen auch in den kleineren Gemeinden der Region, angekommen' ist. Bürgermeister aus Münchens Umlandgemeinden berichteten, dass bezahlbarer Wohnraum auch in ihren Gemeinden ein wichtiges Thema ist und es inzwischen um weit mehr geht als die Errichtung der bisher typischen Einfamilien- und Reihenhäuser. Somit ist - nicht nur in diesem Kontext - Wissen gefragt, wie Menschen wohnen möchten. Und diese Frage ist insbesondere in den Wachstumsräumen in Deutschland besonders virulent.

Dr. Michael Droß

dross@ssr-muenchen.de

1 Schulten Stadt- und Raumentwicklung, Wilhelm-Wagenfeld-Straße 16, 80807 München, Deutschland
Die Studie zu Wohnstandortentscheidungen von Doppelverdienerhaushalten von Rebekka Oostendorp kommt daher zum richtigen Zeitpunkt, stellen doch solche Haushalte inzwischen ein Fünftel aller Haushalte in Deutschland dar, mit wachsender Tendenz. In Doppelverdienerhaushalten sind beide Partner berufstätig, ob jeweils in Teil- oder in Vollzeit ist dabei unerheblich. Sie stehen vor der Herausforderung, drei Orte miteinander zu vereinbaren, nämlich zwei Arbeitsorte mit einem gemeinsamen Wohnstandort. Hintergrund der Untersuchung von Rebekka Oostendorp sind die Veränderungen der Arbeitswelt: die deutliche Zunahme der Frauenerwerbstätigkeit, die Zunahme von befristeten Verträgen, von Projektarbeit und Überstunden. Das bedeutet auch, dass eine wachsende Zahl von Menschen die Bereiche Arbeit, Wohnen und Freizeit sowohl räumlich als auch zeitlich nicht mehr klar unterscheidet. Dies erschwert die raum-zeitliche Organisation des Alltags.

Die Studie ist räumlich im Kontext polyzentrischer Stadtregionen angesiedelt. Es besteht die Annahme, dass deren intensive Verflechtungen vielfältige Möglichkeiten für ihre Bewohnerinnen und Bewohner bieten. In polyzentrischen Stadtregionen stehen an mehreren Orten und in guter Erreichbarkeit eine Vielzahl von Funktionen zur Verfügung. Das dürfte die Alltagsorganisation in Doppelverdienerhaushalten erleichtern. Das Buch ist die Dissertation der Autorin, die im Kontext des DFG-Projekts „Wohnstandortentscheidungen in polyzentrischen Stadtregionen" verfasst wurde. An diesem Projekt waren das Geographische Institut der Universität Bonn, das LeibnizInstitut für Länderkunde in Leipzig und das ILS - Institut für Landes- und Stadtentwicklungsforschung in Dortmund sowie drei Beispielregionen (Köln/Bonn, Östliches Ruhrgebiet und Leipzig/Halle) beteiligt. Die Autorin hat diesen Hintergrund mit großem Gewinn für ein sehr lesenswertes 
Buch genutzt. Die Untersuchungen wurden sehr präzise und sehr anschaulich und nachvollziehbar geführt. So sind die Forschungsfragen sehr gut mit dem Forschungsstand in den jeweiligen Fachgebieten unterfüttert und die Arbeit ist äußerst klar gegliedert. Die Autorin argumentiert sicher und nachvollziehbar.

Die Wohnstandortentscheidungen von Doppelverdienern wurden empirisch anhand der polyzentrischen Stadtregion Köln/Bonn untersucht. Analysiert wurden zum einen die unterschiedlichen Haushalts- und Arbeitssituationen der Doppelverdiener und zum anderen die vielfältigen räumlichen Rahmenbedingungen polyzentrischer Stadtregionen. Die Untersuchung erfolgte in drei Schritten: Zunächst geht es um die Konstellationen von Wohnorten und Arbeitsorten von Doppelverdienerhaushalten in der Region Köln/ Bonn, dann werden die Such- und Entscheidungsprozesse von Doppelverdienerhaushalten bei der Wohnstandortwahl beleuchtet und zuletzt werden die Strategien in Doppelverdienerhaushalten zur Vereinbarung von Wohnen und Arbeiten fokussiert. In der Analyse kommt ein Methodenmix zum Einsatz. Es wurden schriftliche Haushaltsbefragungen aller Haushalte in mehreren Stadtquartieren durchgeführt. Darüber hinaus führte die Autorin qualitative, leitfadenorientierte Interviews in ausgewählten Haushalten. Für die schriftliche Haushaltsbefragung wurden fünf unterschiedlich strukturierte Quartiere in der Region Köln/Bonn ausgewählt, die sich hinsichtlich ihrer Versorgungsqualität und ihrer ÖPNV-Erschließungsqualität unterscheiden. Für die Interviews wurde ebenfalls auf die Unterschiedlichkeit der Haushalte Wert gelegt. Einige der Ergebnisse seien im Folgenden zusammengefasst.

Doppelverdienerhaushalte stellen in allen fünf Quartieren die Mehrheit der erwerbstätigen Haushalte dar. Doppelverdiener wohnen also sowohl in urbanen als auch in zwischenstädtischen Räumen. In den urbanen Quartieren sind aber mehr Doppelverdienerhaushalte festzustellen als am Stadtrand oder in kleineren Orten. Vielfach sind in zwei der untersuchten urbanen Quartiere beide Partner berufstätig und kinderlos, während in den übrigen drei Quartieren Familienhaushalte mit Vollzeit-Teilzeit-Modell überwiegen oder auch ältere Paare ohne Kinder anzutreffen sind. Wenn Familienhaushalte in den urbanen Quartieren wohnen, dann gehen zu einem vergleichsweise hohen Anteil beide Partner in Vollzeit arbeiten. Dies dürfte auf die sehr gute Versorgung und Erschließungsqualität in urbanen Quartieren zurückzuführen sein, welche die Alltagsorganisationen von Familien erheblich erleichtern. Die Ausstattungsqualität des Wohnquartiers gewinnt also für die Doppelverdiener an Bedeutung, die hohen beruflichen Belastungen ausgesetzt sind (Seite 207).

Weiterhin bezieht die Wohnstandortwahl die Entfernung zu den Arbeitsplätzen der Partner ein, stellt diese jedoch nicht unbedingt in den Vordergrund, sondern es spielen auch viele weitere Kriterien eine Rolle. So ist die Ausstattung des Wohnstandorts mit Einrichtungen der Daseinsvorsorge häufig wichtiger als die Nähe zum Arbeitsort. Für den Arbeitsweg bedeutet das jedoch eine hohe Bereitschaft der Haushaltsmitglieder, die guten Verkehrsverbindungen in der Region zu nutzen und längere Wege zurückzulegen (Kapitel 7.3.3).

Die schriftliche Befragung und Interviews zeigen, dass Doppelverdiener häufig vor der Suche genau wissen, wo sie wohnen möchten. Es sind Erfahrungen und Gewohnheiten, Bilder und soziale und emotionale Beziehungen zu bestimmten Orten, die ausschlaggebend sind und nicht etwa ein festgefügter Satz von Kriterien, der an mehrere Standorte in der Region angelegt wird (Seite 174). Die polyzentrische Struktur der Region wird dann berücksichtigt, wenn es um die Erreichbarkeit der Arbeitsorte geht. Doch sind die Vorstellungen vom Wohnen eher lokal geprägt und nicht regional orientiert. Als Vorteil der polyzentrischen Stadtregion stellt die Untersuchung einerseits heraus, dass die Wohn- oder Arbeitssituation auch unabhängig voneinander geändert werden kann, das heißt, Standortentscheidungen haben höhere Freiheitsgrade. Andererseits sorgen die individuellen Präferenzen der Haushaltsmitglieder dafür, dass, obwohl ihre Vorstellungen an mehreren Orten innerhalb der Regionen umgesetzt werden könnten, die Haushalte nicht in der gesamten Region nach einem geeigneten Wohnort suchen (Seite 204). Tendenziell werden weniger Kompromisse beim Wohnen eingegangen als flexible Lösungen im Arbeitsleben gesucht, wie etwa lange Pendelwege, Homeoffice, Selbstständigkeit oder Zweitwohnungen. Dabei sind Frauen mit Teilzeitbeschäftigung häufig wohnortnäher beschäftigt, die Vollzeit-Erwerbstätigkeit des Mannes hat gegenüber der Teilzeit-Erwerbstätigkeit der Frau größeren Einfluss auf die Wohnstandortentscheidung. Lösungen zur Vereinbarkeit von Wohnen und Arbeiten sind bei Frauen in Teilzeit eher auf Kinderbetreuung und Alltagsorganisation ausgerichtet, während Männer Lösungen wie Homeoffice nutzen, um sich beruflich weiter zu entwickeln (Seite 204). Unternehmen sind dazu aufgerufen, ihren Mitarbeiterinnen und Mitarbeitern flexible Lösungen anzubieten. Die Autorin schlägt unter anderem „Dual-Career Services“ vor, wie sie an den großen deutschen Universitäten inzwischen gang und gäbe sind, um auch den Partnerinnen bzw. Partnern zu geeigneten Arbeitsplätzen zu verhelfen (Seite 208).

Aus den Ergebnissen der Arbeit von Rebekka Oostendorp lässt sich schlussfolgern, dass Nutzungsmischung und eine sehr gute öffentliche Verkehrsanbindung der Wohnquartiere eine wichtige Grundlage bilden, um das Alltagsleben angesichts der wachsenden Anforderungen in der Arbeitswelt zu vereinfachen. Insbesondere wohnstandortnahe Kinderbetreuungseinrichtungen sind von zentraler Bedeu- 
tung. Einerseits sollten daher zwischenstädtische Wohnorte entsprechend qualifiziert werden, andererseits sollte die Anziehungskraft städtischer Wohnquartiere gestärkt werden. Dazu gehört es nach Meinung des Rezensenten, die öffentlichen Räume, und das sind vor allem auch Straßenräume, als Lebensräume zurückzugewinnen und zu entwickeln. Kolleginnen und Kollegen, welche sich mit dem Wanderungsverhalten von Haushalten beschäftigen, mit deren Wohn- standortwahl, mit geschlechtsspezifischen Fragestellungen der Wohnstandortwahl, mit Vereinbarungen von Wohnen und Arbeiten und mit raum-zeitlichen Alltagsorganisationen in polyzentrischen Räumen und selbstverständlich allen, die Freude an gut lesbaren und hochwertigen wissenschaftlichen Arbeiten haben, sei das Buch uneingeschränkt zur Lektüre empfohlen. 